\title{
PREVALÊNCIA DE QUEIXAS SUPRA-ESOFÁGICAS EM PACIENTES COM DOENÇAS DO REFLUXO EROSIVA E NÃO-EROSIVA
}

\author{
Gustavo Cálcena AGUERO', Eponina M. 0. LEMME², Ângela ALVARIZ ${ }^{3}$, \\ Beatriz Biccas CARVALHO ${ }^{4}$, Rosana B. SCHECHTER ${ }^{1}$ e Luiz ABRAHÃO Jr. ${ }^{4}$
}

\begin{abstract}
RESUMO - Racional - Na doença do refluxo gastroesofágico pode-se encontrar, como parte do quadro clínico da doença, queixas respiratórias, otorrinolaringológicas, dor torácica ou disfagia. As duas primeiras são intituladas de manifestações supra-esofágicas da doença. É discutível a prevalência destas alterações em pacientes com doença do refluxo gastroesofágico nas formas erosiva e não-erosiva. Objetivos - Avaliar a prevalência de manifestações supra-esofágicas em pacientes com doenças do refluxo erosiva e não-erosiva. Métodos - Foram revistas as fichas de pacientes que realizaram endoscopia digestiva alta, esofagomanometria e pHmetria prolongada nos últimos 5 anos, na investigação de doença do refluxo gastroesofágico (pirose como queixa principal), em que havia informações a respeito de queixas respiratórias e otorrinolaringológicas. Foram selecionados pacientes com doença do refluxo erosiva (graus I a III, pela classificação de Savary-Miller) e com doença do refluxo não-erosiva (endoscopia negativa, com pHmetria prolongada anormal). A análise estatística utilizou o teste de qui-quadrado. Resultados - Duzentos e oitenta pacientes preencheram os critérios de inclusão, sendo 162 com doença do refluxo erosiva ( $70 \%$ com esofagite grau I) e 118 com doença do refluxo não-erosiva. No grupo total, 88 apresentavam queixas otorrinolaringológicas (31\%), com predomínio de rouquidão e pigarro e 42 , queixas respiratórias ( $15 \%$ ), predominando a tosse crônica. Dos pacientes com queixas otorrinolaringológicas, 45 pertenciam ao grupo doença do refluxo erosiva (28\%) e 43 ao grupo doença do refluxo não-erosiva (36,4\%). Em relação aos com queixas respiratórias, 21 pacientes (13\%) pertenciam ao grupo doença do refluxo erosiva e 21 (18\%) ao grupo doença do refluxo não-erosiva. Conclusão - Não houve diferença entre a prevalência das queixas supra-esofágicas em pacientes com doenças do refluxo erosiva e não-erosiva.
\end{abstract}

DESCRITORES - Refluxo gastroesofágico. Transtornos da motilidade esofágica.

\section{INTRODUÇÃO}

A doença do refluxo gastroesofágico (DRGE) é definida como doença crônica relacionada ao fluxo retrógrado do conteúdo gastroduodenal em direção ao esôfago e/ou órgãos adjacentes, resultando em variável espectro de sintomas, com ou sem lesão tecidual ${ }^{(18)}$ A DRGE tem elevada prevalência, sendo que estudos realizados nos EUA demonstram que aproximadamente $18 \%$ de uma população aparentemente saudável refere pirose, o sintoma típico da doença, pelo menos uma vez por semana ${ }^{(15)}$. Recentemente, estudo de prevalência da doença no Brasil, demonstrou por meio de inquérito populacional, que $11,9 \%$ dos quase 14000 indivíduos pesquisados, apresentavam pirose uma ou mais vezes por semana ${ }^{(19)}$.
A DRGE pode ser classificada em doença do refluxo erosiva (DRE), pelo encontro de erosões ou evidências de suas complicações na mucosa esofagiana, na presença de sintomas típicos e doença do refluxo não-erosiva (DRNE) quando existem os mesmos sintomas, porém sem as lesões acima referidas, ao exame endoscópico ${ }^{(3)}$. É importante ressaltar que cerca de $50 \%-70 \%$ dos pacientes que procuram auxílio médico têm DRNE, também denominados de pacientes endoscopicamente negativos ${ }^{(3)}$.

Os sintomas clássicos da DRGE são a pirose e a regurgitação, intitulados de sintomas típicos. Existem também as manifestações atípicas como dor torácica, sintomas respiratórios e otorrinolaringológicos, os dois últimos considerados manifestações supra-esofágicas, uma vez que são provocadas pelo efeito do conteúdo gástrico refluído em regiões que ultrapassam o esôfago ${ }^{(21)}$.

Serviço de Gastroenterologia, Hospital Universitário Clementino Fraga Filho, Universidade Federal do Rio de Janeiro, RJ.

1 Universidade Gama Filho ${ }^{2}$ Departamento de Clínica Médica da Faculdade de Medicina da Universidade Federal do Rio de Janeiro. ${ }^{3}$ Faculdade de Medicina da Universidade Federal do Rio de Janeiro. ${ }^{4}$ Serviço de Gastroenterologia do Hospital Universitário Clementino Fraga Filho da Universidade Federal do Rio de Janeiro, RJ.

Correspondência: Dra. Eponina M. O. Lemme - Trav. Santa Terezinha, 14 - Tijuca - 20271-070 - E-mail: eponina@hucff.ufri.br 
O objetivo deste trabalho foi avaliar a prevalência das manifestações supra-esofágicas em pacientes com DRE e DRNE.

\section{MÉTODOS}

\section{Pacientes}

Entre 1999 e 2004, foram realizadas 2020 esofagomanometrias e 919 pHmetrias prolongadas. Foram revistas as fichas de pacientes submetidos a esses exames na investigação de DRGE e que apresentavam pirose como queixa principal (sensação de queimação retrosternal ascendente em direção à região cervical), associada a informações a respeito de queixas respiratórias (asma iniciada na idade adulta, tosse crônica na ausência de tabagismo, infecção respiratória de repetição, episódios de asfixia noturna) e otorrinolaringológicas (ORL) (rouquidão freqüente, pigarro, dor de garganta e sensação de globo). Todos haviam sido submetidos previamente a endoscopia digestiva alta (EDA).

Foram selecionados pacientes com DRE (presença de esofagite pela classificação de Savary-Miller) ${ }^{(24)}$ e com DRNE (sem esofagite, mas com pHmetria prolongada anormal). Pacientes com estenose esofágica ou esôfago de Barrett não foram incluídos. Também não o foram os pacientes com diagnóstico de DRNE que tivessem feito uso de antisecretores no período de, pelo menos, 1 mês precedendo o exame endoscópico.

\section{Métodos}

\section{Endoscopia digestiva alta}

Todos os pacientes haviam sido submetidos a endoscopia digestiva alta, com a técnica habitual, após sedação consciente. Esofagite, quando presente, foi descrita segundo a classificação de Savary-Miller ${ }^{(24)}$ (grau I - erosões isoladas, grau II - erosões confluentes porém não-circunferenciais, grau III - erosões confluentes e circunferenciais). Hérnia hiatal foi definida pelo achado da junção esofagogástrica $2 \mathrm{~cm}$ ou mais acima do pinçamento diafragmático.

\section{pHmetria esofagiana prolongada}

Foram empregados aparelhos Synetics MK II e MK III, utilizando-se cateter com um sensor de antimônio posicionado a $5 \mathrm{~cm}$ do limite superior do esfíncter inferior do esôfago (EIE), previamente localizado pela esofagomanometria, realizada com técnica convencional ${ }^{(13)}$. Considerou-se episódio de refluxo a queda do $\mathrm{pH}$ esofagiano abaixo de 4 durante, pelo menos, 15 segundos. Os detalhes de técnica foram previamente descritos ${ }^{(11)}$. Inibidores de bomba protônica foram suspensos por 7-10 dias previamente ao exame, inibidores $\mathrm{H} 2$ por 48 72 horas e procinéticos 24 horas antes. O paciente permanecia por um período de 24 horas com o equipamento, durante o qual era orientado a se alimentar de forma o mais natural possível (evitando a ingesta de alimentos cítricos e bebidas do tipo cola), preenchendo relatório com relação aos horários das refeições, posição corporal e registro dos sintomas. Considerou-se refluxo anormal quando a percentagem de tempo total de $\mathrm{pH}$ $<4$ situava-se acima de $4,5 \%{ }^{(7)}$.

\section{Análise dos dados}

A análise estatística utilizou o teste do qui-quadrado e o valor de $P<0,05$ para significância. Foram comparados os dados de prevalência das queixas supraesofágicas (ORL e respiratórias) nos grupos DRE e DRNE e em relação ao grau de esofagite.

\section{RESULTADOS}

Duzentos e oitenta pacientes preencheram os critérios de inclusão, sendo 162 com DRE ( $58 \%$ do sexo masculino, média de idade 49 anos) e 118 com DRNE (63\% do sexo feminino, média de idade 47 anos). Os dados demográficos de ambos os grupos estão na Tabela 1 .

TABELA 1. Dados demográficos dos pacientes $(n=280)$

\begin{tabular}{lcc}
\hline & DRE $(\mathrm{n}=162)$ & DRNE $(\mathrm{n}=118)$ \\
\hline Masc/fem (\%) & $58 / 42$ & $37 / 63$ \\
Idade (média em anos) & 49 & 47 \\
\hline
\end{tabular}

DRE $=$ doença do refluxo erosiva

A hérnia de hiato estava presente em 141/162 pacientes com DRE (87\%) e em 77/118 (65\%) dos com DRNE.

Entre os 280 pacientes, 88 apresentavam queixas otorrinolaringológicas (31\%) (Tabela 2) e 42, queixas respiratórias (15\%) (Tabela 3$)$, alguns apresentando mais de um sintoma.

TABELA 2. Queixas otorrinolaringológicas $(\mathrm{n}=88)$

\begin{tabular}{lc}
\hline Queixas & $\mathbf{n}(\%)$ \\
\hline Rouquidão & $50(51 \%)$ \\
Pigarro & $38(38 \%)$ \\
Dor de garganta & $07(7 \%)$ \\
Globo & $04(4 \%)$ \\
Total de queixas & $99(100 \%)$ \\
\hline
\end{tabular}

TABELA 3. Queixas respiratórias $(\mathrm{n}=42)$

\begin{tabular}{lc}
\hline Queixas & $\mathbf{n}(\%)$ \\
\hline Tosse & $35(68,6 \%)$ \\
Asma & $6(11,7 \%)$ \\
Asfixia noturna & $6(11,7 \%)$ \\
Infecção respiratória de repetição & $4(8 \%)$ \\
Total de queixas & $51(100 \%)$ \\
\hline
\end{tabular}

Dos pacientes com queixas ORL, 45 pertenciam ao grupo DRE (28\%) e 43 ao grupo DRNE (36,4\%). Em relação aos com queixas respiratórias, 21 pacientes $(13 \%)$ pertenciam ao grupo DRE e 21 (18\%) ao grupo DRNE. Não houve diferença significativa entre a prevalência das queixas supra-esofágicas nos dois grupos de pacientes (Tabela 4). A maioria dos pacientes do grupo DRE apresentava esofagite grau I, sem diferença significativa em relação às queixas (Tabela 5). 
TABELA 4. Prevalência das queixas supra-esofágicas $(\mathrm{n}=280)$

\begin{tabular}{|c|c|c|c|}
\hline & DRE $(n=162)$ & DRNE $(\mathrm{n}=118)$ & Valor de $P$ \\
\hline ORL $(\mathrm{n}=88)$ & $45(28 \%)$ & $43(36,4 \%)$ & 0,12 \\
\hline $\operatorname{RESP}(\mathrm{n}=42)$ & $21(13 \%)$ & $21(18 \%)$ & 0,26 \\
\hline \multicolumn{4}{|c|}{$\begin{array}{l}\text { ORL = queixas otorrinolaringológicas } \\
R E S P=\text { queixas respiratórias } \\
\text { DRE = doença do refluxo erosiva } \\
\text { DRNE = doença do refluxo não-erosiva }\end{array}$} \\
\hline \multicolumn{4}{|c|}{ TABELA 5. Grupo DRE - grau de esofagite x queixas } \\
\hline Esofagite & ORL & RESP & $P$ valor \\
\hline Grau I & $34(75,6 \%)$ & $15(71,4 \%)$ & 0,7 \\
\hline Grau II & $9(20 \%)$ & $6(28,6 \%)$ & 0,4 \\
\hline Grau III & $2(4,4 \%)$ & - & - \\
\hline
\end{tabular}

$\mathrm{ORL}=$ queixas otorrinolaringológicas

DRE $=$ doença do refluxo erosiv

\section{DISCUSSÃo}

A DRGE é uma das doenças mais comuns que afetam o trato gastrointestinal e que, por vezes, pode gerar sintomas em outros órgãos além do esôfago. Denomina-se refluxo extraesofágico (refluxo esôfago-laringo-faringeo, esôfago-brônquico) quando o conteúdo refluído ultrapassa o esôfago, provocando manifestações percebidas em cavidade oral, faringe, laringe e sistema respiratório ${ }^{(27)}$. Em uma grande série $(\mathrm{n}=1511)$ foi demonstrado que, pelo menos, uma manifestação atípica estava presente em $80 \%$ dos pacientes que se apresentavam com sintomas típicos de refluxo ${ }^{(15)}$. Estudo caso-controle envolvendo 101366 pacientes internados encontrou risco aumentado de várias manifestações extra-esofagianas, principalmente asma, pneumonia e fibrose pulmonar em portadores de esofagite ou estenose de esôfago ${ }^{(2)}$.

Deve-se ressaltar também que, grande parte dos pacientes que apresentam manifestações extra-esofágicas da DRGE como queixa principal, não refere os sintomas clássicos ou típicos. Isto é relatado em 57\%-94\% dos pacientes com manifestações laríngeas, em 43\%-75\% dos com tosse crônica e em 40\%-60\% dos pacientes com asma, supostamente relacionadas à $\mathrm{DRGE}^{(28)}$.

Entre as manifestações respiratórias, estão descritas a asma iniciada na idade adulta, tosse crônica na ausência de tabagismo, bronquite crônica, fibrose pulmonar idiopática e pneumonias. Como parte das manifestações otorrinolaringológicas, são citadas desde alterações anatômicas como laringite posterior, ulcerações de contato, granulomas, estenose e carcinoma da laringe, até sintomas como rouquidão, pigarro, dor de garganta, globo faríngeo, tosse e otalgia ${ }^{(21)}$. A presença desses sintomas pressupõe como possíveis mecanismos fisiopatológicos um efeito nocivo direto do suco gástrico sobre a árvore traqueo-brônquica, laringo-faringe, ouvido médio, complexo nasosinusal e/ou ativação de reflexos vagais pelo refluxo do conteúdo gástrico na porção distal do esôfago, resultando em hiperreatividade brônquica $^{(21,28)}$. Muitas vezes não é possível diferenciar se a DRGE é realmente o fator causal das manifestações extraesofagianas ou se há associação de condições patogênicas que são bastante prevalentes na população em geral ${ }^{(2)}$. Entretanto, sua relação causal com a DRGE encontra substrato na melhora de muitos pacientes tratados com potentes drogas anti-secretoras que inibem a acidez gástrica ${ }^{(23)}$.

Para que ocorra a DRGE é necessária a existência de alguns mecanismos:1) fraqueza do EIE, atribuída pelo menos em parte, a anormalidades nos mecanismos reguladores dependentes de óxido nítrico $(\mathrm{ON})$ e que também atuam na defesa da mucosa do esôfago, predispondo-a a lesão ${ }^{(14)}$; 2) presença de hérnia de hiato que, além de possibilitar o refluxo do ácido contido no saco herniário para o esôfago através do EIE, por fraqueza deste ou por abertura forçada pela pressão intra-pleural negativa, dificulta o clareamento adequado de ácido pelo esôfago ${ }^{(16)}$; 3 ) aumento do número e duração dos relaxamentos transitórios do EIE, que são o principal mecanismo responsável pelos episódios de refluxo ${ }^{(20)}$.

Os mecanismos envolvidos na gênese do refluxo esofagiano proximal ainda não estão totalmente esclarecidos. Alguns foram propostos, tais como a incompetência do $\mathrm{EIE}^{(25)}$, os próprios relaxamentos transitórios ${ }^{(17)}$ e a deficiência de motilidade do corpo esofagiano $^{(9)}$. A motilidade esofagiana ineficaz, a alteração motora mais prevalente na DRGE, tem sido encontrada em pacientes com manifestações respiratórias e refluxo comprovado ${ }^{(4)}$.

Episódios de refluxo gastroesofagiano proximal são mais freqüentes em pacientes com sintomas atípicos de DRGE do que em indivíduos saudáveis ${ }^{(6)}$, com episódios de relaxamento transitório do EEI pouco presentes nos refluidores em esôfago proximal em relação aos que se apresentavam com refluxo distal. Isto sugere envolvimento de outros mecanismos na extensão proximal do refluxo, tais como o volume de ácido que passa pelo EIE ou a velocidade elevada do refluxo ao longo da luz do esôfago ${ }^{(6)}$.

Em análise multivariada observou-se que o sexo feminino, idade avançada, gravidade, duração da doença do refluxo e fumo são fatores de risco para a ocorrência de alterações extraesofagianas, o mesmo não ocorrendo com a obesidade ${ }^{(21)}$. Esta é reconhecidamente fator de risco isolado para sintomas de DRGE e desenvolvimento de esofagite ${ }^{(1)}$.

É de particular interesse reconhecer algumas diferenças entre DRE e DRNE. A primeira está relacionada a níveis elevados de acidificação do esôfago. Estudos pHmétricos demonstram que o percentual de tempo total de exposição ao $\mathrm{pH}<4$ é significativamente maior em pacientes com DRE do que nos com DRNE, diferença esta que se mantém em relação aos tempos de exposição ácida em posição supina ${ }^{(5)} \mathrm{e} e \mathrm{em}$ ambas as posições supina e ortostática ${ }^{(12)}$. A média da pressão do EIE é menor na DRE em relação aos portadores de $\mathrm{DRNE}^{(5)}$, assim como há maior prevalência de hipotensão do $\operatorname{EIE}^{(12)}$, de hérnia hiatal ${ }^{(5,10)}$ e de alterações motoras ${ }^{(9)}$.

No presente estudo, as manifestações ORL mais freqüentes foram a rouquidão e o pigarro e entre as respiratórias, houve franco predomínio da tosse crônica, resultados semelhantes ao referido na literatura ${ }^{(8)}$. Demonstrou-se que não houve diferença na prevalência entre os grupos DRE e DRNE, quanto a presença de queixas supra-esofágicas, tanto as otorrinolaringológicas, como as respiratórias. Embora de caráter retrospectivo, os critérios de seleção incluíram apenas os pacientes em cujas fichas, registradas no próprio laboratório de realização de exames manométrico 
e pHmétrico, havia referência à presença das queixas supraesofágicas (com sua caracterização) ou a sua ausência. Além disso, o número de pacientes é bastante expressivo, com adequada distribuição entre os portadores de DRE e DRNE ${ }^{(3)}$.

A literatura não apresenta muitos trabalhos explorando o tema objeto deste estudo. JASPERSEN et al. ${ }^{(8)}$ estudaram prospectivamente 6215 pacientes com sintomas sugestivos de DRGE. Desses, $52 \%$ foram classificados como DRE e $48 \%$ como DRNE. Manifestações por eles intituladas de extra-esofágicas, foram detectadas em $32,8 \%$ de todos os pacientes, $34,9 \%$ no grupo DRE e 30,5\% no grupo DRNE, leve mas estatisticamente mais prevalentes no primeiro. Neste estudo incluiu-se também a dor torácica não-cardíaca, que foi a manifestação mais freqüente $(14,5 \%)$, seguida pela tosse crônica (13\%), alterações laríngeas $(10 \%)$ e asma $(4,8 \%)$. Quando se compararam apenas as manifestações respiratórias e ORL, as diferenças foram pequenas e os próprios autores sugeriram que elas poderiam estar ampliadas pelo grande tamanho da amostra. Entretanto, resultados semelhantes foram observados em número menor de pacientes: sintomas supra-esofágicos, foram significativamente mais prevalentes em pacientes com DRE que DRNE ( $\mathrm{n}=157$; $57 \%$ x $33 \%-P<0,001)^{(22)}$. Outro estudo comparando 50 pacientes com esofagite e sintomas típicos, com 50 controles sem essas características, mostrou manifestações atípicas em $66 \%$ dos pacientes versus $42 \%$ dos controles $(P=0,02)^{(26)}$. Sintomas supra-esofágicos foram mais freqüentes em pacientes com DRNE e pHmetria alterada do que em portadores de pirose funcional (sintomáticos, com pHmetria normal) - $72 \%$ de 25 pacientes $\mathrm{X}$ $62 \%$ de 18 pacientes ${ }^{(29)}$.

É importante assinalar que mais de $70 \%$ de pacientes desta série apresentavam esofagite leve (grau I), sem diferença em relação à queixa apresentada. No estudo anteriormente citado $^{(8)}$, quando se comparou a ocorrência das queixas entre os pacientes com esofagite leve (graus A e B de Los Angeles) e pacientes com DRNE, as diferenças não foram significativas, sugerindo a possibilidade de que pacientes com esofagite leve, se comportem de maneira semelhante aos pacientes sem esofagite (DRNE). É possível que, em parte, este fato possa justificar os resultados aqui apresentados.

\section{CONCLUSÃO}

Não foram encontradas diferenças entre a prevalência das queixas supra-esofágicas em pacientes com doenças do refluxo erosiva e não-erosiva. Há necessidade de mais estudos para avaliar a real prevalência e significado destas manifestações nos dois grupos de pacientes, assim como os mecanismos responsáveis pelas mesmas.

Aguero GC, Lemme EMO, Alvariz A, Carvalho BB, Schechter RB, Abrahão Jr L. Prevalence of supraesophageal manifestations in patients with gastroesophageal erosive and non-erosive reflux disease. Arq Gastroenterol. 2007;44(1):39-43.

ABSTRACT - Background - Respiratory, ear-nose and throat complaints, chest pain and dysphagia can be a part of clinical manifestations of gastroesophageal reflux disease. The first two are named supraesophageal manifestations of gastroesophageal reflux disease. Controversy about the prevalence of these clinical manifestations in patients with non-erosive and erosive gastroesophageal reflux disease exists. Aims - Evaluate the prevalence of supraesophageal manifestations in patients with erosive and non-erosive gastroesophageal reflux disease. Methods - Files from patients submitted to upper endoscopy, esophageal manometry and $\mathrm{pH}$ monitoring for the investigation of gastroesophageal reflux disease (heartburn as the chief complaint) were reviewed and respiratory and ear, nose and throat symptoms were recorded. Patients with erosive disease (grades I to III according to Savary-Miller classification) and with non-erosive disease (normal endoscopy with abnormal pH monitoring were selected. Statistical analysis included the chi-square test. Results - Two hundred and eighty patients fulfilled the inclusion criteria being 162 with erosive disease (70\% with grade I esophagitis) and 118 with non-erosive disease. Overall, 88 patients had ear, nose and throat symptoms (31\%), the more frequent were hoarseness and clearing and 42 , respiratory manifestations (15\%), being cough the more prevalent. In the ear, nose and throat symptoms group, 45 were erosive disease (28\%) and 43 non-erosive disease (36.4\%). As for the respiratory symptom group, 21 patients (13\%) were erosive disease and $21(18 \%)$ were non-erosive disease. Conclusion - There was no difference in the prevalence of supraesophageal manifestations between patients with gastroesophageal erosive and non-erosive reflux disease.

HEADINGS - Gastroesophageal reflux. Esophageal motility disorders. 


\section{REFERÊNCIAS}

1. El-Serag HB, Graham DY, Satia JA, Rabeneck l. Obesity is an independent risk factor for GERD symptoms and erosive esophagitis. Am J Gastroenterol. 2005;100:1243-50.

2. El-Serag HB, Sonnemberg A. Comorbid occurrence of laryngeal or pulmonary disease with esophagitis in United States military veterans. Gastroenterology. 1997;113:755-60.

3. Fass R, Brien F, Nimish VL. Non erosive reflux disease. Current concepts and dilemmas Am J Gastroenterol. 2001;96:303-14.

4. Fouad YM, Katz PO, Hatlebakk IG, Castell DO. Ineffective esophageal motility: the most common motility abnormality in patients with GERD-associated respiratory symptoms. Am J Gastroenterol. 1999;94:1464-7.

5. Frazzoni M, De Micheli E, Zenhlin P, Savarino P. Pathophysiological characteristics of patients with non-erosive reflux disease differ from those of patients with functional heartburn. Aliment Pharmacol Ther. 2004;20:81-8.

6. Grossi I, Cicaglione AF, Marzio I. Transient lower oesophageal sphincter relaxation play an insignificant role in gastro-oesophageal reflux to the proximal oesophagus Neurogastroenterol Motility. 2001;13:503-9.

7. Jamieson IR, Stein HJ, De Meester TR, Bonavina L, Schwizer W, Hinder RA, Albertucc M. Ambulatory 24-hour esophageal $\mathrm{pH}$ monitoring: normal values, optimal threshold, specificity, sensitivity and reproducibility. Am J Gastroenterol. 1992;87:1102-11.

8. Jaspersen D, Kulig M, Labenz J, Leodolter A, Lind T, Meyer-Sabelleck W, Vieth M, Willich SN, Lindner D, Stolte M, Malfertheiner P. Prevalence of extra-esophagea manifestations in gastro-oesophageal reflux disease: an analysis based on the ProGERD study. Alim Pharm Ther. 2003;17:1515-20.

9. Kasapida P, Xynos E, Mantides A. Differences in manometry and 24-h ambulatory pH-metry between patients with and without endoscopic or histological esophagitis in gastroesophageal reflux disease. Am J Gastroenterol. 1993;88:1893-9.

10. Lemme EMO, Abrahão Jr LJ, Manhães Y, Schechter R, Carvalho BB, Alvariz A. Ineffective esophageal motility in gastroesophageal erosive reflux disease and in non erosive reflux disease. Are they different? J Clin Gastroenterol. 2005;39:224-7.

11. Lemme EMO, Almeida SM, Firman CMG, Pantoja JA, Nascimento FAP. pHmetria esofagiana prolongada - avaliação de 170 exames. Arq Gastroenterol. 1997;34:71-6.

12. Lemme EMO, Domingues GR, Abrahão Jr LJ. Gastroesophageal reflux, lowe esophageal sphincter pressure and innefective esophageal motility in Barrett's esophagus: a comparative study [abstract]. Dis Esophagus. 2001;14 (suppl):AO93.

13. Lemme EMO, Domingues G, Silva LFD, Firman CMG, Pantoja JA. Esofagomanometria computadorizada - resultados preliminares em voluntários saudáveis. GED Gastroenterol Endosc Dig. 2001;20:29-35.

14. Liu B. Effects of nitric oxide synthase inhibitor to esophagus in a feline esophagitis model. Dis Esophagus. 2003;16:312-4.
15. Locke GR, Talley NI, Fett SL, Zinsmeister AR, Melton LJ. Prevalence and clinical spectrum of gastroesophageal reflux: a population based study in Olmstead County, Minnesota. Gastroenterology. 1997;112:1448-56

16. Mittal RK, Balaban DH. Mechanisms of disease: the esophagogastric junction. N Engl J Med. 1997;336:924-32.

17. Mittal RK, Holloway RH, Penagini R, Blackshaw LA, Dent J. Transient lower esophageal sphincter relaxation. Gastroenterology. 1995;109:601-10.

18. Moraes-Filho JPP, Cecconello I, Gama-Rodrigues J, Castro LP, Henry MA, Meneghelli UG, Quigley E. Brazilian consensus on gastroesophageal reflux disease: proposals for assessment, classification, and management. Am J Gastroenterol. 2002;97:241-8.

19. Moraes-Filho JPP, Chinzon D, Eisig JN, Hashimoto CL, Zaterka S. Prevalência da pirose e da doença do refluxo gastroesofágico na população urbana brasileira. Arq Gastroenterol. 2005;42:122-7.

20. Nam QN, Holloway R Gastroesophageal reflux disease. Curr Opin Gastroenterol. 2003;19:373-8.

21. Poelmans J, Feenstra L, Demedts J, Rutgeerts P, Tack J. The yield of upper gastrointestinal endoscopy in patients with suspected reflux-related chronic ear, nose and throat symptoms. Am J Gastroenterol. 2004;99:1419-26.

22. Rahia J, Hietanem E, Soureander L. Symptoms of gastroesophageal reflux disease in elderly people. Age Ageing. 1991;5:365-70.

23. Richter JE. Extraesophageal manifestations of gastro-oesophageal reflux disease. Aliment Pharmacol Ther. 2005;22(suppl.1):70-80.

24. Savary M, Miller G. The esophagus. Handbook and atlas of endoscopy. Solothurn, Switzerland: Verlag Grassmann; 1978. p.135-42.

25. Sloan S, Radmaker AW, Kahrilas PJ. Determinants of gastroesophageal junction incompetence: hiatal hernia, lower esophageal sphincter or both? Ann Intern Med. 1992;117:977-82

26. Suazo J, Facha MT, Valdovinos MA. Case and control study of atypical manifestations in gastroesophageal reflux disease. Rev Invest Clin. 1998;50:317-22.

27. Tauber S, Gross M, Issing WJ. Association of laryngopharyngeal symptoms with gastroesophageal reflux disease. Laryngoscope. 2002;12:879-86.

28. Wong WM, Fass R. Extraesophageal and atypical manifestations of GERD. J Gastroenterol Hepatol. 2004;19:s33-s43.

29. Ylitalo R, Indestad P, Hertergard S. Pharyngeal and laryngeal symptoms and signs related to extraesophageal reflux in patients with heartburn in gastroenterology practice: a prospective study. Clin Otolaryngol. 2005;30:347-52. 